\title{
Asociación entre desenlaces clínicos y cumplimiento del protocolo de recuperación mejorada después de la cirugía (ERAS) en procedimientos colorrectales: estudio multicéntrico
}

\author{
Association between clinical outcomes and compliance with the enhanced \\ recovery after surgery (ERAS) protocol in colorectal procedures: \\ a multicenter study
}

Fredy Orlando Mendivelso ${ }^{1} \mathbb{D}$, Arnold José Barrios-Parra² ${ }^{\mathbb{D}}$, Eduardo Zárate-López ${ }^{3}$, Ángela María Navas-Camacho ${ }^{4} \mathbb{D}$, Adrián Osvaldo Álvarez ${ }^{5} \mathbb{D}$, Santiago Mc Loughlin ${ }^{5} \mathbb{D}$,

Gabriela Renee Mendoza ${ }^{5}$, Daniel Enciso-Pérez ${ }^{6}$, Rubén Rodríguez-Barajas ${ }^{7}$, María Sofía Jiménez-Chávez ${ }^{8} \mathbb{D}$, José Ramírez ${ }^{7} \mathbb{D}$, Florencia Faber ${ }^{9} \mathbb{D}$, Gonzalo Solla ${ }^{10}$, Marcelo Viola-Malet ${ }^{11}$, Milena Rodríguez-Bedoya ${ }^{12}$

1 MD., MPH., MSc., especialista en Epidemiología, FETP (Field Epidemiology Training Program), Clínica Reina Sofía, Bogotá, D.C., Colombia.

Médico, especialista en Cirugía general, Jefe del Departamento Quirúrgico. Clínica Colsanitas, Bogotá D.C., Colombia.

Médico, especialista en Anestesiología, jefe del Departamento de Anestesiología, Clínica Reina Sofía, Bogotá, D.C., Colombia.

Médico, especialista en Cirugía general, Departamento de Cirugía, Clínica Reina Sofía, Bogotá, D.C., Colombia.

Médico, especialista en Anestesiología, Departamento de Anestesia, Hospital Italiano, Buenos Aires, Argentina.

Médico, especialista en Cirugía general, Hospital Civil de Guadalajara, México.

Médico, especialista en Anestesiología, Hospital Civil de Guadalajara, México.

Magistra en Ciencias de Enfermería, Hospital Civil de Guadalajara, México.

Médico, especialista en Anestesiología, Centro de Asistencia Médica del Oeste, CAMOC, Uruguay.

10 Médico, especialista en Anestesiología, Corporación de Asistencia Médica (MUCAM), Uruguay.

11 Médico, especialista en Cirugía general, Corporación de Asistencia Médica (MUCAM), Uruguay.

12 Médica Veterinaria, especialista en Epidemiología clínica, docente Facultad de Medicina, Fundación Universitaria Sanitas, Bogotá, D.C., Colombia.

\section{Resumen}

Introducción. Los protocolos de recuperación mejorada después de cirugía se han diseñado como una innovación en salud tras demostrarse que la mejora en los dispositivos médicos y la depuración de técnicas alcanzaron la meseta en disminución de complicaciones. Con estas estrategias de la medicina perioperatoria, en cirugía colorrectal se reducen la morbilidad y estancia hospitalaria. El objetivo del estudio fue evaluar si la tasa de adherencia al programa de recuperación mejorada después de la cirugía (ERAS) está asociada con los resultados quirúrgicos.

Fecha de recibido: 16/05/2020 - Fecha de aceptación: 13/07/2020

Correspondencia: Fredy Orlando Mendivelso, Calle 127 \# 20-78, Clínica Reina Sofía, Bogotá, D.C., Colombia. Teléfono: 3043844102. Correo electrónico: fmendivelso@colsanitas.com

Citar como: Mendivelso FO, Barrios-Parra AJ, Zárate-López E, Navas-Camacho AM, Álvarez AO, Mc Loughlin S, Mendoza GR, et al. Asociación entre desenlaces clínicos y cumplimiento del protocolo de recuperación mejorada después de la cirugía (ERAS) en procedimientos colorrectales: estudio multicéntrico. Rev Colomb Cir. 2020;35:601-13. https://doi.org/10.30944/20117582.662

Este es un artículo de acceso abierto bajo una Licencia Creative Commons - BY-NC-ND https://creativecommons.org/licenses/by-ncnd/4.0/deed.es 
Métodos. Estudio multicéntrico, observacional, retrospectivo de cohorte (2015-2019), en cinco hospitales latinoamericanos certificados por la sociedad ERAS. Se calculó la incidencia de complicaciones quirúrgicas durante el posquirúrgico inmediato (30 días) y la duración de la estancia hospitalaria. Se utilizaron análisis bivariado y regresión logística multivariada para evaluar los factores asociados con la tasa de complicaciones.

Resultados. Fueron incluidos en el estudio 648 pacientes en cinco hospitales ERAS, con edad promedio de 6I años y mayor porcentaje de hombres (5I \%). El cumplimiento global al protocolo ERAS fue de $75 \%$ y la estancia promedio de 6,2 días (mediana: 4 días). Se tuvo un cumplimiento óptimo del protocolo ERAS (igual o mayor al $80 \%$ ) en $23,6 \%$ de los pacientes. Se documentó fuga de la anastomosis en $4 \%$, complicaciones infecciosas en $8,4 \%$, íleo en 5,7 \%, reingreso en IO,2 \% y mortalidad de I,I \%. El análisis multivariado mostró que los niveles de adherencia óptima al protocolo ERAS reducen significativamente la aparición de complicaciones como fuga de

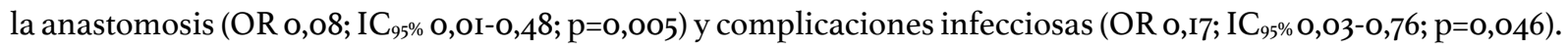

Discusión. Los resultados sugieren que un cumplimiento del programa ERAS mayor al $80 \%$ se asocia a menor frecuencia de complicaciones en pacientes con cirugía electiva colorrectal.

Palabras clave: recuperación mejorada después de la cirugía; enfermedades del colon; cirugía colorrectal; medidas de asociación, exposición, riesgo o desenlace; protocolos clínicos; cumplimiento y adherencia al tratamiento.

\section{Abstract}

Introduction. Enhanced Recovery After Surgery (ERAS) protocol has been designed as an innovation in health after demonstrating that the improvement in medical devices and the refinement of techniques reached the plateau in reducing complications. With these strategies of perioperative medicine, in colorectal surgery morbidity and hospital stay are reduced. The aim of the study was to evaluate whether the rate of adherence to the ERAS protocol is associated with surgical outcomes.

Methods. Multicenter, observational, retrospective cohort study (2015-2019), in five Latin American hospitals certified by the ERAS Society. The incidence of surgical complications during the immediate postoperative period (30 days) and length of hospital stay were calculated. Bivariate analyzes and multivariate logistic regression were used to assess factors associated with complication rates.

Results. 648 patients were included in the study in five ERAS hospitals, with an average age of 6I years and a higher percentage of men (5I\%). Overall compliance with the ERAS protocol was $75 \%$ and the average stay was 6.2 days (median: 4 days). There was optimal compliance with the ERAS protocol (equal to or greater than $80 \%$ ) in $23.6 \%$ of the patients. Anastomotic leak was documented in $4 \%$, infectious complications in $8.4 \%$, ileus in $5.7 \%$, readmission in I0.2\%, and mortality in $\mathbf{1 . 1} \%$. Multivariate analysis showed that optimal adherence levels to the ERAS protocol significantly reduce the appearance of complications such as anastomotic leakage (OR 0.08 ; $95 \% \mathrm{CI}$ 0.0I-0.48; $\mathrm{p}=0.005$ ) and infectious complications (OR 0.I7; 95\% CI 0.03-0.76; $\mathrm{p}=0.046$ ).

Discussion. The results suggest that compliance with the ERAS program greater than $80 \%$ is associated with a lower frequency of complications in patients with elective colorectal surgery.

Keywords: enhanced recovery after surgery; colonic diseases; colorectal surgery; measures of association, exposure, risk or outcome; clinical protocols; treatment adherence and compliance.

\section{Introducción}

La evolución de la cirugía colorrectal históricamente ha estado ligada al conocimiento anatómico, al perfeccionamiento de la técnica quirúrgica y al desarrollo tecnológico de mejores equipos. La mortalidad ha pasado del $50 \%$ al inicio del siglo XX a un 4,6\% en la actualidad ${ }^{\mathrm{I}-3}$, en relación con mejoras en la detección temprana, la quimioterapia y las medidas de soporte. Todas estas medidas de cuidado perioperatorio han demostrado disminución de morbilidad y mortalidad quirúrgicas, las cuales se optimizan 
con la generación de estrategias de intervención multimodal, conocidas como protocolo ERAS (del inglés Enhanced Recovery after Surgery) ${ }^{4}$. Las complicaciones, quirúrgicas y no quirúrgicas, aumentan los costos y la demanda de servicios, teniendo un impacto directo en pacientes, familiares y sistema de salud ${ }^{5,6}$.

El protocolo ERAS propone un abordaje multidisciplinario, diseñado para disminuir y contrarrestar el efecto deletéreo que ocasiona el catabolismo secundario a la respuesta metabólica del organismo ante el trauma quirúrgico, para optimizar al paciente en sus valores fisiológicos basales (figura I). Los resultados se traducen en tasas reducidas de morbilidad, menor número de complicaciones relacionadas con la atención en salud, recuperación más rápida y menor estancia hospitalaria ${ }^{4-12}$.

El protocolo ERAS contiene varios elementos, formulados con la mejor evidencia científica, que se distribuyen para su aplicación en la vía clínica del paciente, desde el preoperatorio mediato e inmediato hasta el trans y posquirúrgico, haciendo énfasis en la modulación del estrés quirúrgico $^{12}$. Un equipo multidisciplinario, conformado por cirujano, anestesiólogo, enfermera, nutricionista y terapeuta físico, se encarga de optimizar la adherencia a cada una de las estrategias ${ }^{\mathrm{I}}$.

En cirugía colorrectal hay 24 recomendaciones incluidas en el manejo del paciente que va a ser llevado a cirugía electiva:

\section{Preadmisión}

I. Cese del consumo de cigarrillo y de alcohol excesivo

2. Evaluación nutricional preoperatoria y, según sea necesario, evaluación y soporte nutricional

3. Optimización del tratamiento y control de las enfermedades basales crónicas

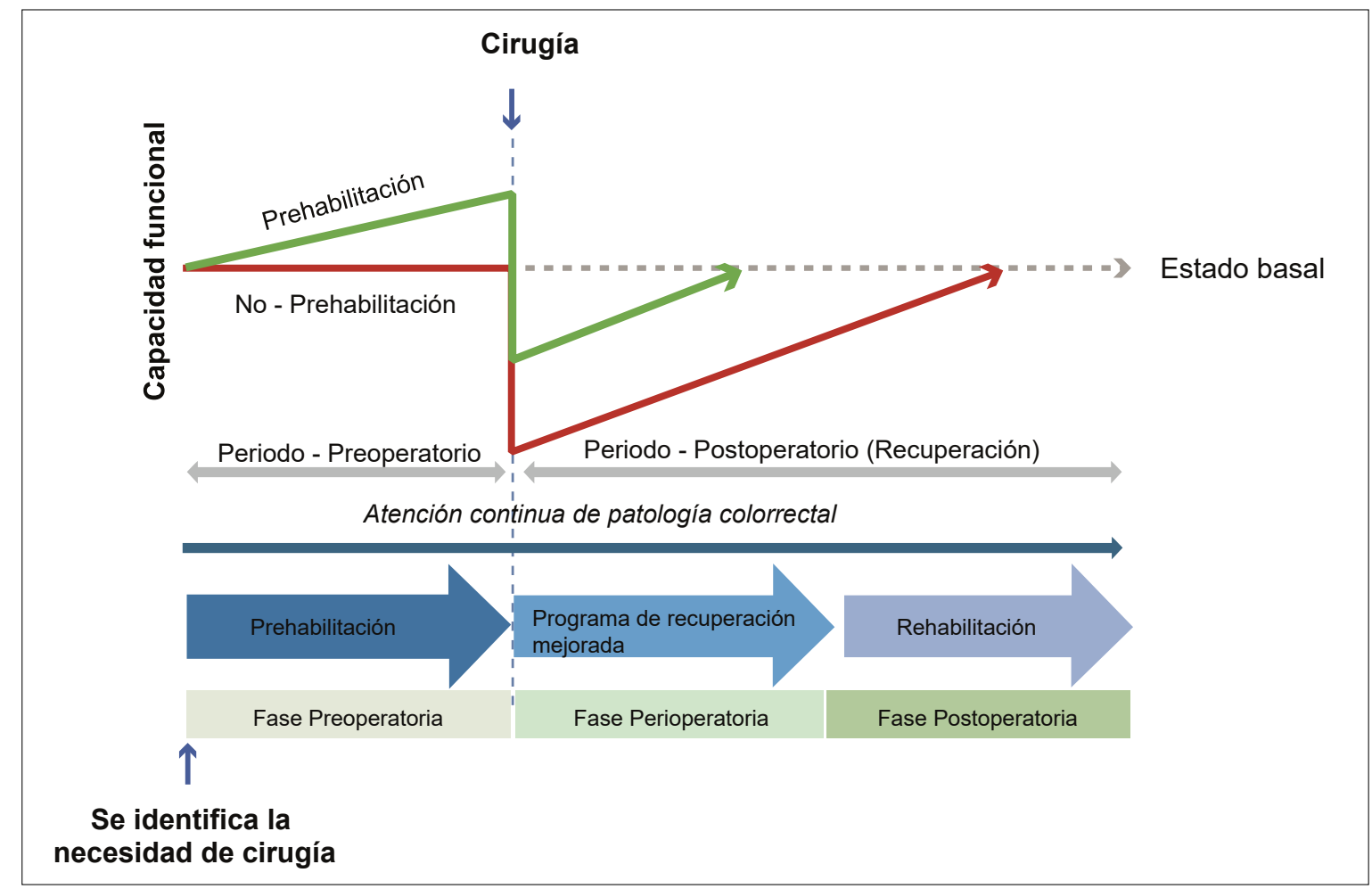

Figura 1. La prehabilitación multimodal mejora la capacidad funcional antes y después de la cirugía colorrectal Fuente: elaborado por los autores. 


\section{Preoperatorio}

4. Información preoperatoria estructurada y compromiso real del paciente y familiares o cuidadores, haciéndolos participes y corresponsables de la recuperación

5. Disminución del tiempo de ayuno

6. Profilaxis preoperatoria contra la trombosis

7. Profilaxis preoperatoria contra la infección

8. Profilaxis contra las náuseas y vómitos

\section{Intraoperatorio}

9. Propender por la cirugía mínimamente invasiva, sin excluir a la cirugía por vía abierta

I0. Anestesia estandarizada, evitando opioides de larga duración

II. Mantener la euvolemia

I2. Anestesia epidural para cirugía abierta

I3. Uso restrictivo de los drenajes del sitio quirúrgico

I4. Extracción de tubos nasogástricos antes de la reversión de la anestesia

I5. Control de la temperatura corporal utilizando mantas de flujo de aire caliente e infusiones intravenosas calentadas

\section{Posquirúrgico}

I6. Movilización precoz (día de la cirugía)

I7. Ingesta temprana de líquidos y sólidos orales (ofrecido el día de la cirugía)

I8. Extracción temprana de catéteres urinarios y fluidos intravenosos (mañana después de la cirugía)

19. Uso de café, goma de mascar, laxantes y agentes bloqueadores de opioides periféricos (cuando se usan opioides)

20. Ingesta de proteínas y suplementos nutricionales ricos en energía
2I. Abordaje multimodal para el control del dolor ahorrador de opioides

22. Abordaje multimodal para el control de náuseas y vómitos

23. Prepararse para el alta temprana

24. Auditoría periódica de resultados y procesos con el equipo multidisciplinario del programa ERAS ${ }^{13}$

La estandarización de las estrategias modulan la complejidad del procedimiento y minimizan la respuesta al estrés que rodea el procedimiento ${ }^{14-18}$. Múltiples estudios han reportado que un cumplimiento de las estrategias mayor al $70 \%$ se traduce en disminución de la tasa de complicaciones hasta un $50 \%{ }^{12}$.

La más reciente revisión sistemática y metaanálisis de los protocolos de recuperación mejorada en cirugía colorrectal electiva en adultos, tanto abierta como laparoscópica, concluye que respecto a los protocolos ERAS, si bien mejoran los resultados de los pacientes sin aumentar los eventos adversos, no existe suficiente evidencia para determinar qué o cuáles componentes, o combinaciones de componentes de la estrategia de cuidado multidisciplinar, son clave para mejorar los resultados del paciente ${ }^{19}$. Contrario a estos resultados, otros autores cuestionan que no es necesario tener altos porcentajes de adherencia al protocolo para lograr resultados quirúrgicos adecuados ${ }^{20}$.

A diferencia de otras regiones, los protocolos ERAS en cirugía colorrectal se han implementado recientemente en países latinoamericanos y la evaluación del impacto, experiencia y resultados de la implementación ha sido poco documenta$\mathrm{da}^{21}$. El objetivo del estudio fue medir el grado de adherencia a las estrategias de intervención de un programa de excelencia clínica en cirugía y evaluar si la tasa de adherencia al programa ERAS está asociada con los desenlaces clínicos vistos, como los resultados quirúrgicos y el momento del alta. 


\section{Métodos}

\section{Población}

Estudio retrospectivo de cohorte, con pacientes adultos (edad igual o mayor a I8 años) llevados a cirugía colorrectal electiva, durante el periodo 20152019. El trabajo de investigación fue desarrollado en cinco hospitales latinoamericanos certificados y auditados internacionalmente por la Sociedad ERAS Internacional como Centros de Excelencia en la implementación del programa y protocolos de manejo quirúrgico de patología colorrectal [Clínica Reina Sofía, Bogotá, D.C., Colombia; Hospital Italiano de Buenos Aires, Argentina; Hospital Civil de Guadalajara, México; Médica Uruguaya Corporación de Asistencia Médica (MUCAM), Montevideo y Centro de Asistencia Médica del Oeste de Colonia (CAMOC), Uruguay].

Tras su entrenamiento y certificación como centros de excelencia ERAS, los hospitales incluidos en la investigación comenzaron a implementar el protocolo ERAS en diferentes momentos durante el periodo analizado, lo cual significa claramente que tienen diferentes volúmenes de práctica y que por conexión directa obligó a la selección de este diseño de cohorte.

El tamaño de la muestra fue probabilístico y calculado con el método de Westland ajustado por pérdidas y relacionado directamente con el número de parámetros a incluir en los modelos multivariados planteados. Un muestreo aleatorio simple con reemplazo se realizó con los registros del sistema de auditoría interactivo basado en web (ERAS - Interactive Audit System $\left.^{\circledR}\right)$. El cálculo de una muestra probabilística y una técnica de muestreo aleatorio son la alternativa más eficiente en esta cohorte de naturaleza no concurrente (es decir, que todos los sujetos del estudio no entran en la misma fecha al estudio) y que para efectos del análisis de los datos todas las observaciones pudieran ser llevadas a tiempo cero $\left(t_{o}\right)$, disminuyendo el sesgo de cohorte que acompaña este tipo de diseños.

Se recolectó información individual sobre variables clínicas, antecedentes médico-quirúr- gicos, procedimientos de cirugía, anestesia y de todas las intervenciones establecidas en el protocolo ERAS para cirugía colorrectal.

Los criterios de exclusión fueron: manejo quirúrgico de urgencia (por sangrado, obstrucción aguda o perforación), pacientes con tumores colorrectales con criterio de inoperabilidad, resección multivisceral planificada, y paciente remitido de otras instituciones para realizar el procedimiento quirúrgico de colon sin vinculación previa al protocolo desde la etapa de prehabilitación.

\section{Análisis estadístico}

Las variables cuantitativas se analizaron mediante medidas de frecuencia, tendencia central y dispersión; los datos categóricos, mediante proporciones; los supuestos de distribución normal en las variables de interés, con la prueba no paramétrica de Shapiro-Wilk; la correlación entre variables cuantitativas, con el coeficiente Rho de Pearson, y los análisis bivariados, con el estadístico de chi $^{2}$ para independencia o la prueba exacta de Fisher ${ }^{22}$. La comparación de variables cuantitativas se hizo con las pruebas estadísticas $t$ student o $\mathrm{U}$ de Mann Whitney. En el contraste de hipótesis se consideraron estadísticamente significativos valores de $\mathrm{p}<0,05$.

Para determinar la asociación entre el porcentaje de adherencia al protocolo ERAS se establecieron cuatro niveles: baja (o \% a $59 \%$ ), aceptable (60 \% a $69 \%$ ), buena (70 \% a $79 \%$ ) y óptima ( $80 \%$ a IOo \%). Estos niveles se compararon con la tasa de complicaciones mediante un modelo multivariado de regresión logística.

Se incluyeron como variables de confusión la edad, sexo, índice de masa corporal (IMC), puntaje de la clasificación funcional ASA, diagnóstico de cáncer colorrectal, total de líquidos intravenosos administrados durante la intervención, tiempo de cirugía, enfoque quirúrgico (abierto vs. laparoscópico), técnica anastomótica, grupo del procedimiento (recto vs. colon e intestino delgado), administración de opioides en cirugía, uso 
de drenes en sitio de la resección y prescripción de AINES durante el posquirúrgico.

Para los desenlaces de interés se reportaron medidas de asociación (Odds Ratio: OR), tanto univariadas como multivariadas, acompañados de su correspondiente intervalo de confianza del $95 \%\left(\mathrm{IC}_{95 \%}\right)$. La posibilidad de interacción no fue considerada y la multicolinealidad entre variables fue descartada mediante el cálculo del factor de inflación de varianza (solo incluidos VIF $<$ IO), parar disminuir los errores estándar en los modelos. Todos los datos se analizaron con el programa Stata (StataCorp, College Station, TX, USA) V.I5.

\section{Desenlaces}

La principal medida de desenlace fue la presencia de fuga anastomótica dentro de los primeros 30 días después de la cirugía. Otros desenlaces analizados fueron complicaciones infecciosas e íleo paralítico posquirúrgico.

Tabla 1. Características generales de los pacientes incluidos en el estudio

\begin{tabular}{lcc}
\hline Variable & $\mathbf{n}$ & $\%$ \\
\hline Edad (años) mediana y RIQ & 63 & 21,5 \\
Grupo de edad (años) & & \\
$\quad<65$ & 356 & 54,9 \\
$\quad \geq 65$ & 292 & 45,1 \\
Sexo & & \\
$\quad$ Mujer & 317 & 48,9 \\
$\quad$ Hombre & 331 & 51,1 \\
Fumador & & \\
$\quad$ No & 567 & 87,6 \\
$\quad$ Sí & 72 & 11,1 \\
$\quad$ Interrupción por cirugía & 8 & 1,2 \\
Consumo de alcohol & & \\
$\quad$ No & 618 & 95,4 \\
$\quad$ Sí & 26 & 4,0 \\
$\quad$ Interrupción por cirugía & 4 & 0,6 \\
Antecedentes médicos & & \\
$\quad$ Diabetes mellitus & 66 & 10,2 \\
Enfermedades cardíacas graves & 25 & 3,9 \\
$\quad$ Enfermedades pulmonares graves & 26 & 4,0 \\
IMC (Kg/m²) mediana y RIQ & 25,6 & 6,2 \\
Quimioterapia preoperatoria & 74 & 11,4 \\
Antecedente de cirugía abdominal & 303 & 46,8 \\
\hline
\end{tabular}

RIQ: Rango intercuartílico $\left(Q_{3}-Q_{2}\right)$; IMC: Índice de masa corporal

\section{Resultados}

Entre los años 2015 y 2019 se recolectó y analizó información de 648 pacientes intervenidos por patología quirúrgica colorrectal en cinco instituciones latinoamericanas con acreditación internacional por la Sociedad ERAS. Poco más de la mitad de los pacientes eran hombres (5I \%), la mediana de edad fue de 63 años y los adultos mayores representaron el $45 \%$ (tabla I). Se encontró antecedente de consumo de tabaco en II \% y consumo habitual de alcohol en $4 \%$. La enfermedad crónica no trasmisible más frecuente fue

Tabla 2. Características relacionadas con la cirugía, la anestesia y el desenlace en los pacientes incluidos en el estudio

\begin{tabular}{|c|c|c|}
\hline Variable & $\mathbf{n}$ & $\%$ \\
\hline $\begin{array}{l}\text { Paciente con enfermedad oncológica } \\
\text { colorrectal (benigna o maligna) }\end{array}$ & 436 & 67,3 \\
\hline Tiempo quirúrgico (minutos) mediana y RIQ & 180 & 110 \\
\hline Estancia hospitalaria (días) mediana y RIQ & 4 & 4 \\
\hline Estancia en UCl (días) mediana y RIQ & 4 & 4 \\
\hline \multicolumn{3}{|l|}{ Profilaxis de la trombosis } \\
\hline Sin profilaxis & 216 & 33,3 \\
\hline Anticoagulante (heparina o HBPM) & 75 & 11,6 \\
\hline $\begin{array}{l}\text { Combinación de anticoagulante y } \\
\text { compresión }\end{array}$ & 357 & 55,1 \\
\hline \multicolumn{3}{|l|}{ Enfoque quirúrgico } \\
\hline Laparoscopia estándar & 404 & 62,4 \\
\hline Cirugía abierta & 132 & 20,4 \\
\hline Abordaje a través de estoma existente & 74 & 11,4 \\
\hline Laparoscópica asistida a mano & 28 & 4,3 \\
\hline $\begin{array}{l}\text { Conversión (Laparoscópica a cirugía } \\
\text { abierta) }\end{array}$ & 10 & 1,5 \\
\hline \multicolumn{3}{|l|}{ Clasificación funcional ASA } \\
\hline I & 66 & 10,2 \\
\hline II & 443 & 68,4 \\
\hline III & 118 & 18,2 \\
\hline IV & 6 & 0,9 \\
\hline Sin información & 15 & 2,3 \\
\hline Ingreso a UCI durante estancia principal & 69 & 10,7 \\
\hline Muerte & 7 & 1,1 \\
\hline \multicolumn{3}{|l|}{ Porcentaje de adherencia al protocolo ERAS } \\
\hline $0 \%-59 \%$ & 154 & 23,8 \\
\hline $60 \%-69 \%$ & 164 & 25,3 \\
\hline $70 \%-79 \%$ & 176 & 27,2 \\
\hline $80 \%-100 \%$ & 154 & 23,8 \\
\hline
\end{tabular}

RIQ: Rango intercuartílico $\left(Q_{3}-Q_{2}\right)$; UCl: Unidad de cuidados intensivos; HBPM: Heparina de bajo peso molecular; ASA: Clasificación funcional de la Sociedad Americana de Anestesiología 
Diabetes mellitus (Io \%), el $47 \%$ de los pacientes tenían cirugía abdominal previa y ir \% recibieron quimioterapia en los últimos 6 meses.

En $67 \%$ de los pacientes operados presentaban enfermedad oncológica colorrectal (benigna o maligna) (tabla 2). La profilaxis antitrombótica se administró en $66 \%$ de los pacientes. Los procedimientos quirúrgicos realizados con mayor frecuencia fueron la hemicolectomía derecha (23\%) y la izquierda (I6\%). El abordaje quirúrgico preferido por los cirujanos fue laparoscópico (66\%). La mediana de tiempo quirúrgico fue de I8o minutos. El io \% de los pacientes fueron trasladados a UCI y la mediana de estancia en UCI fue de 4 días. Finalmente, Io,2 \% de los pacientes reingresaron y la mortalidad en la cohorte fue de I,I \%.

La adherencia al protocolo ERAS en las cinco instituciones fue en promedio del $75 \%$ y en el $23 \%$ de los pacientes se registraron niveles óptimos de adherencia (igual o mayor al 80 \%).
La mediana de estancia hospitalaria en la cohorte fue de 4 días, sin embargo, fue mayor (8 días) en el grupo de pacientes con adherencia baja (menor al 6o \%) y menor (3 días) en el grupo de pacientes con adherencia óptima (igual o mayor al 8o \%) (Kruskal-Wallis $\mathrm{p}=0,008$ ) (figura 2). El estadístico Rho de Pearson también presentó una correlación negativa entre los porcentajes de adherencia y la duración de la estancia hospitalaria $(\rho=-0,42)$.

En los análisis bivariados (tabla 3), los niveles de adherencia óptima presentaron menor tiempo quirúrgico (mediana=I55 minutos, $\mathrm{p}=0,000$ ), menor volumen de líquidos administrados durante el procedimiento (mediana $=800 \mathrm{cc}, \mathrm{p}=\mathrm{0}, 000$ ) y menor estancia hospitalaria (mediana $=3$ días, $\mathrm{p}=0,000$ ). La frecuencia de complicaciones quirúrgicas también fue menor $(7,8 \%, \mathrm{p}=0,002)$, al igual que las infecciosas $(2,6 \%, p=0,003)$. Finalmente, el ingreso a UCI fue muy bajo en pacientes con adherencia óptima $(2 \%, p=0,000)$.

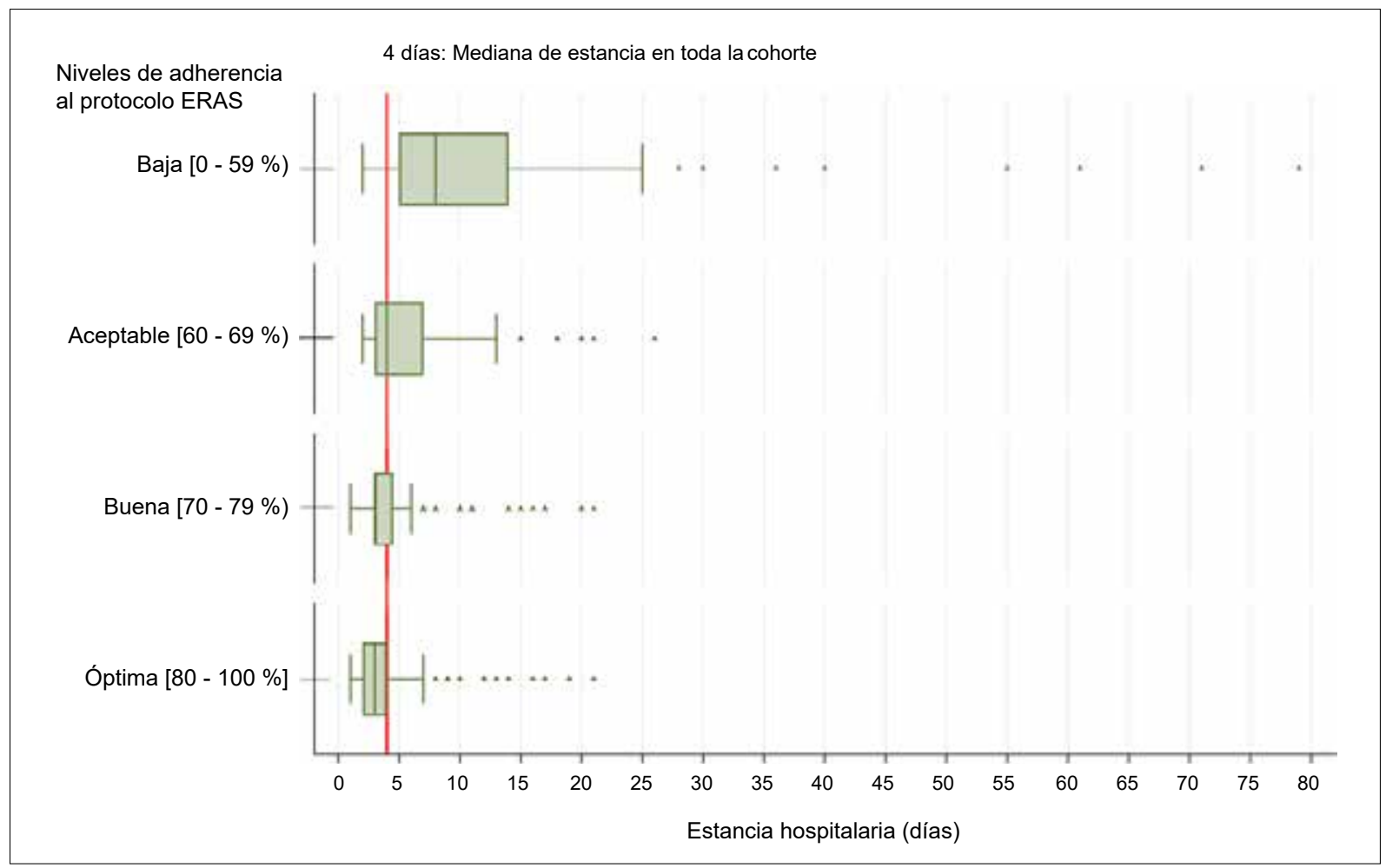

Figura 2. Adherencia al protocolo ERAS y su relación con la estancia hospitalaria 
Tabla 3. Comparación entre niveles óptimos de adherencia al protocolo ERAS, características de la intervención y complicaciones durante el posquirúrgico en cirugía colorrectal

\begin{tabular}{|c|c|c|c|}
\hline \multirow{3}{*}{ Variable } & \multicolumn{2}{|c|}{ Adherencia total } & \multirow{3}{*}{$\mathrm{p}$ valor } \\
\hline & $<80 \%$ & $\geq 80 \%$ & \\
\hline & $(n=494)$ & $(n=154)$ & \\
\hline IMC $\left(\mathrm{Kg} / \mathrm{m}^{2}\right)$, mediana (RIQ) & $25,3(6,6)$ & $26,3(5,4)$ & $0,193^{* *}$ \\
\hline Tiempo quirúrgico (minutos), mediana (RIQ) & $190(125)$ & $155(76)$ & 0,000 ** \\
\hline Total de líquidos endovenosos durante la cirugía (cc), mediana (RIQ) & $1100(1200)$ & $800(470)$ & 0,000 ** \\
\hline Pérdida de sangre intraoperatoria (cc), mediana (RIQ) & $50(250)$ & $150(250)$ & 0,000 ** \\
\hline Estancia en UCl (días), mediana (RIQ) & $4(4)$ & $3(5)$ & $0,513^{* *}$ \\
\hline Estancia hospitalaria (días), mediana (RIQ) & $4(5)$ & $3(2)$ & 0,000 ** \\
\hline Estancia durante el reingreso (días), mediana (RIQ) & $14(11)$ & $7,5(8)$ & $0,037^{* *}$ \\
\hline \multicolumn{4}{|l|}{ Tipo de complicación } \\
\hline Quirúrgicas, n (\%) & $90(18,3)$ & $12(7,8)$ & 0,002 \\
\hline Renales, hepáticas, pancreáticas y gastrointestinales, n (\%) & $73(14,8)$ & $10(6,5)$ & 0,007 \\
\hline Infecciosas, n (\%) & $51(10,3)$ & $4(2,6)$ & 0,003 \\
\hline Respiratorias, n (\%) & $17(3,4)$ & - & 0,020 * \\
\hline Cardiovasculares, n (\%) & $17(3,5)$ & - & 0,010 * \\
\hline Dolor, n (\%) & $16(3,3)$ & $1(0,7)$ & 0,059 * \\
\hline Psiquiátricas, n (\%) & $6(1,2)$ & - & 0,194 * \\
\hline Relacionadas con la anestesia epidural o intradural, n (\%) & $2(0,4)$ & - & 0,585 * \\
\hline Anestésicas, n (\%) & $2(0,4)$ & . & 0,585 * \\
\hline Ingreso a UCI, n (\%) & $66(13,4)$ & $3(2,0)$ & 0,000 \\
\hline Readmisión durante el posquirúrgico, n (\%) & $52(10,5)$ & $14(9,1)$ & 0,607 \\
\hline
\end{tabular}

RIQ: Rango intercuartílico $\left(Q_{3}-Q_{2}\right)$; IMC: Índice de masa corporal; UCI: Unidad de Cuidados Intensivos $\left.{ }^{* *}\right)$ Prueba de suma de rangos de Wilcoxon.

$\left.{ }^{*}\right)$ Prueba exacta de Fisher cuando no se cumplieron los supuestos para poder realizar la prueba Chi2.

\section{Fuga de la anastomosis}

Se presentaron 26 casos de fuga de la anastomosis durante los primeros 30 días de posquirúrgico $(26 / 648=4 \%)$. Incorporando los principales predictores al modelo logístico multivariado $\left(\mathrm{R}^{2}=0,22\right)$, se identificaron tres predictores como factores de protección para la aparición de fuga: uso del dren en la zona de resección (OR o,30; $\left.\mathrm{IC}_{95 \%} \mathrm{0}, 09-0,98 ; \mathrm{p}=0,048\right)$, adherencia al protocolo ERAS buena (OR 0,I3; $\mathrm{IC}_{95 \%}$ 0,03-0,57; $\mathrm{p}=0,007$ ) y adherencia óptima (OR o,o8; $\mathrm{IC}_{95 \%} \mathrm{O}, \mathrm{OI}-\mathrm{O}, 48$; $\mathrm{p}=0,005)$ (figura 3 ).

\section{Complicaciones infecciosas}

En 55 pacientes se presentaron complicaciones infecciosas $(55 / 648=8,49 \%)$. El modelo multiva- riado final $\left(\mathrm{R}^{2}=\mathrm{O}, \mathrm{I} 7\right)$ identificó tres factores que disminuyen el riesgo de complicaciones infecciosas en el posquirúrgico: cirugía por vía laparoscópica (OR o,33; IC 95\% $_{9}$ O,I3-0,84; p=O,O2I), adherencia al protocolo ERAS buena (OR, 0,27; $\left.\mathrm{IC}_{95 \%}, 0,07-0,95 ; \mathrm{p}=0,042\right)$ y adherencia óptima (OR o,I7; $\mathrm{IC}_{95 \%} \mathrm{O}, \mathrm{O} 3-0,76$; $\mathrm{p}=0,046$ ). La anastomosis entre intestino delgado y colon (OR II,6; IC ${ }_{95} \%$ I,24-I27,99; $\mathrm{p}=0,03 \mathrm{I})$ fue la variable más relacionada con la aparición de complicaciones infecciosas (figura 4).

\section{Íleo paralítico posquirúrgico}

El íleo como complicación afectó a 37 pacientes en el posquirúrgico $(37 / 648=5,73 \%)$. El modelo multivariado $\left(\mathrm{R}^{2}=0,09\right)$ no identificó variables des- 


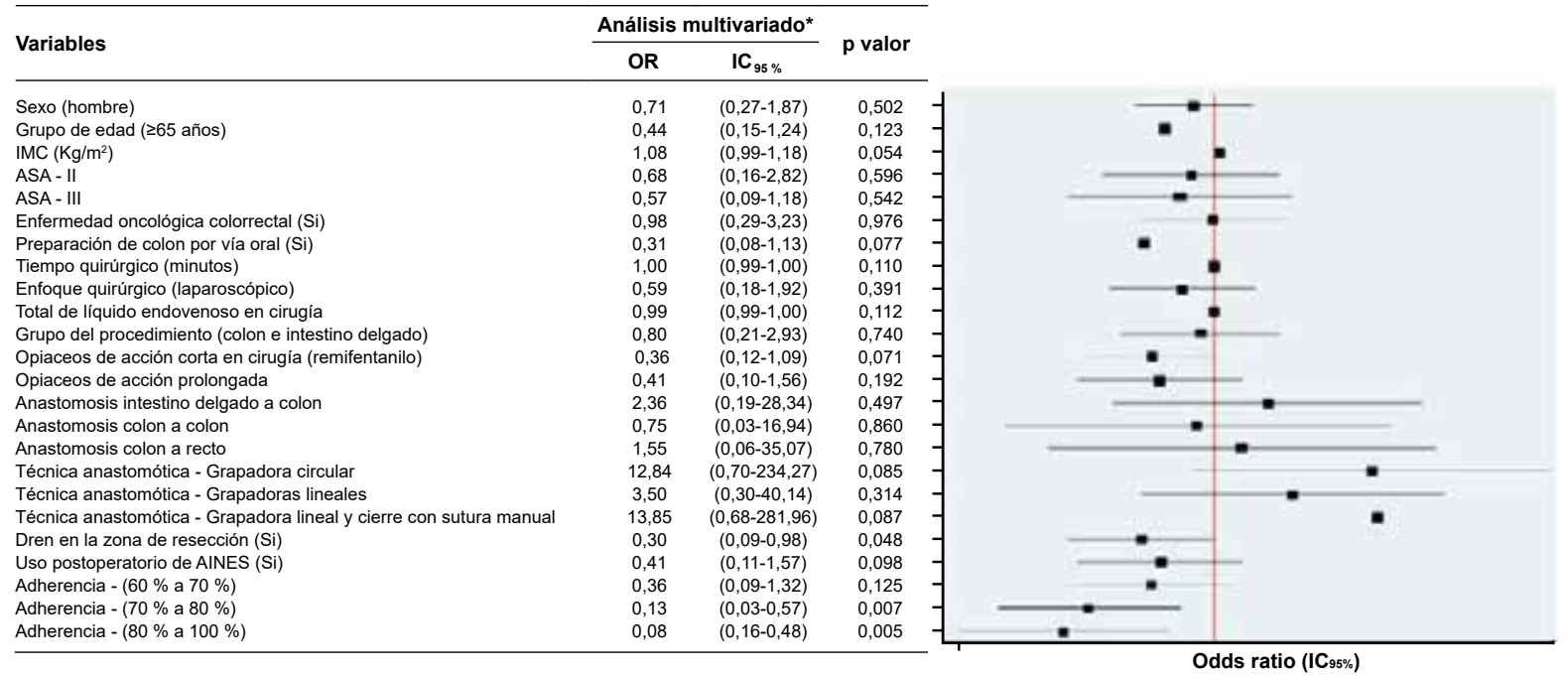

Figura 3. Factores asociados con la aparición de fuga de la anastomosis como complicación en cirugía electiva colorrectal con protocolo ERAS. Estudio multicéntrico $(n=648)$

\begin{tabular}{|c|c|c|c|}
\hline \multirow{2}{*}{ Variables } & \multicolumn{2}{|c|}{ Análisis multivariado* } & \multirow{2}{*}{ p valor } \\
\hline & OR & $I_{95} \%$ & \\
\hline Sexo (Hombre) & 0,31 & $(0,62-2,75)$ & 0,467 \\
\hline Grupo de edad (265 años) & 0,61 & $(0,28-1,33)$ & 0,219 \\
\hline IMC $\left(K g / \mathrm{m}^{2}\right)$ & 1,06 & $(0,99-1,13)$ & 0,085 \\
\hline ASA - II & 1,35 & $(0,33-5,42)$ & 0,672 \\
\hline ASA - III & 1,19 & $(0,24-5,78)$ & 0,826 \\
\hline Enfermedad oncológica colorrectal (Si) & 1,2 & $(0,44-3,27)$ & 0,719 \\
\hline Preparación de colon por vía oral (Si) & 0,50 & $(0,19-1,30)$ & 0,158 \\
\hline Tiempo quirúrgico (minutos) & 1,00 & $(0,99-1,00)$ & 0,841 \\
\hline Enfoque quirúrgico (laparoscópico) & 0,33 & $(0,13-0,84)$ & 0,021 \\
\hline Total de líquido endovenoso en cirugía & 1,00 & $(0,99-1,00)$ & 0,212 \\
\hline Grupo del procedimiento (colon e intestino delgado) & 0,46 & $(0,15-1,42)$ & 0,181 \\
\hline Opiaceos de acción corta en cirugía (remifentanilo) & 0,54 & $(0,23-1,26)$ & 0,156 \\
\hline Opiaceos de acción prolongada & 0,93 & $(0,31-2,74)$ & 0,900 \\
\hline Anastomosis intestino delgado a colon & 11,6 & $(1,24-107,99)$ & 0,031 \\
\hline Anastomosis con reservorio ileoanal (IPAA) & 12,41 & $(0,37-408,15)$ & 0,158 \\
\hline Anastomosis colon a colon & 5,16 & $(0,55-48,24)$ & 0,150 \\
\hline Anastomosis colon a recto & 4,95 & $(0,41-59,28)$ & 0,207 \\
\hline Anastomosis Coloanal & 16,67 & $(0,80-345,16)$ & 0,069 \\
\hline Anastomosis multiples & 12,31 & $(0,69-216,86)$ & 0,086 \\
\hline Técnica anastomótica - Grapadora circular & 1,12 & $(0,30-4,11)$ & 0,858 \\
\hline Técnica anastomótica - Grapadoras lineales & 0,72 & $(0,20-2,54)$ & 0,620 \\
\hline Técnica anastomótica - Grapadora lineal y cierre con sutura manual & 0,81 & $(0,08-7,73)$ & 0,860 \\
\hline Dren en la zona de resección (Si) & 0,38 & $(0,14-1,01)$ & 0,054 \\
\hline Uso postoperatorio de AINES (Si) & 0,77 & $(0,22-2,62)$ & 0,682 \\
\hline Adherencia - $(60 \%$ a $70 \%)$ & 0,63 & $(0,23-1,76)$ & 0,385 \\
\hline Adherencia - $(70 \%$ a $80 \%)$ & 0,27 & $(0,07-0,95)$ & 0,042 \\
\hline Adherencia - ( $80 \%$ a $100 \%)$ & 0,17 & $(0,03-0,76)$ & 0,046 \\
\hline
\end{tabular}

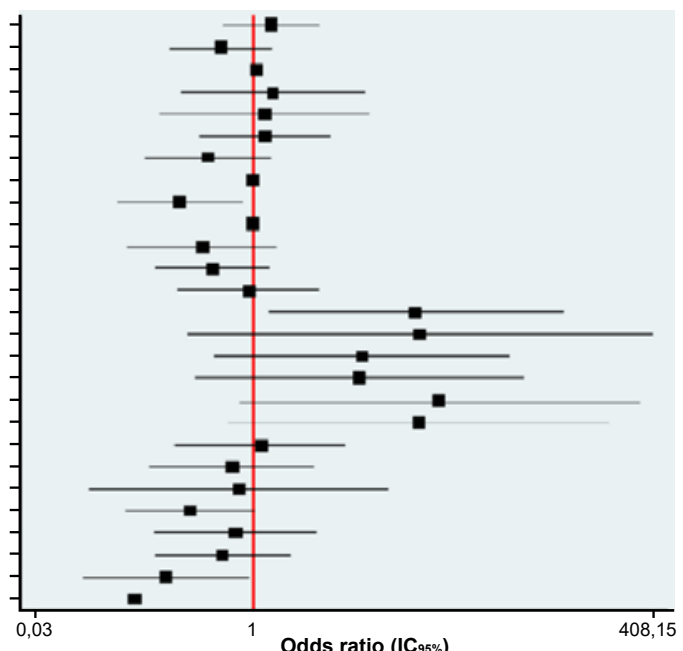

Figura 4. Factores asociados con la aparición de complicaciones infecciosas en cirugía electiva colorrectal con protocolo ERAS. Estudio multicéntrico $(n=648)$

de el punto de vista estadístico que pudieran ser consideradas como un factor asociado al riesgo de presentar íleo durante el posquirúrgico (figura 5).

\section{Discusión}

Los protocolos ERAS para cirugías de alta complejidad le asignan al indicador de estancia hospitalaria durante el posquirúrgico un valor importante, como la mejor forma de evaluar los resultados de su implementación, dado que a estancias cortas, menor número de complicaciones, tanto de naturaleza médica como quirúrgica, y rápida recuperación de las funciones del tracto gastrointestinal en el caso de patología colorrectal 13,23,24. La implementación de ERAS en cirugía electiva de colon y recto ha favorecido los desenlaces en pacientes que requieren este tipo de intervención. En la cohorte analizada, la me- 
diana de la estancia hospitalaria fue de 4 días y disminuye a 3 días con adherencias superiores al $80 \%$. Se encontró una correlación negativa entre los niveles de adherencia al protocolo ERAS y la duración de la estancia hospitalaria ${ }^{25}$.

Hubo mayor incidencia de complicaciones con adherencias menores al $60 \%$. Aunque algunos estudios han descrito que buenos resultados pueden obtenerse con adherencias bajas al protocolo ${ }^{20}$, los resultados de nuestra investigación mostraron que con adherencias iguales o superiores al $80 \%$, la incidencia de complicaciones, la estancia hospitalaria y otros desenlaces negativos disminuyen significativamente, indicando una clara relación de dosis-respuesta ${ }^{26,27}$. La incidencia de fuga la anastomosis en el estudio (4\%) fue similar a la reportada por otros estudios multicéntricos ${ }^{28-32}$.

Otros estudios reportaron que la falta de terapia de fluidos equilibrada (OR 3,8), la falta de movilización temprana (OR 20,7), el uso prolongado de catéter urinario $(\mathrm{OR} 4,5)$ y el uso de drenes (OR 2,8) se asociaron significativamente con estancias prolongadas $19,20,32,33$.

Este estudio mostró que, independientemente de condiciones basales de los pacientes llevados a cirugía de colon y recto, como tener o no una enfermedad oncológica o poseer una condición de fragilidad fisiológica como la que puede encontrarse en el adulto mayor, finalmente fue el nivel de cumplimiento a las estrategias del protocolo ERAS el principal indicador para alcanzar mejores resultados en salud y corta estancia hospitalaria, y sigue siendo el atributo al que se le concede mayor valor en las instituciones con protocolo ERAS, lo cual coincide con los hallazgos descritos ampliamente en la literatura ${ }^{34,35}$.

Las complicaciones esperadas en un procedimiento quirúrgico son determinadas por el grado de complejidad que acompaña la cirugía y la pericia del cirujano, así como las condiciones basales del paciente, sus enfermedades y grado de control, el compromiso sistémico que pueda causar el estadio de una enfermedad oncológica o crónica degenerativa y otros múltiples factores susceptibles de ser identificados, y en algunos casos, intervenidos o modificados. Para todas las situaciones resulta en extremo importante seguir pautas de optimización en preacondicionamiento clínico o prehabilitación, con el propósito de lograr mejores desenlaces en salud, tanto para el paciente como para las mismas instituciones que manejan este tipo de procedimientos ${ }^{20,36-39}$.

Pocos estudios han evaluado y fijado una propuesta sobre el número necesario de procedimientos de cirugía colorrectal para que los

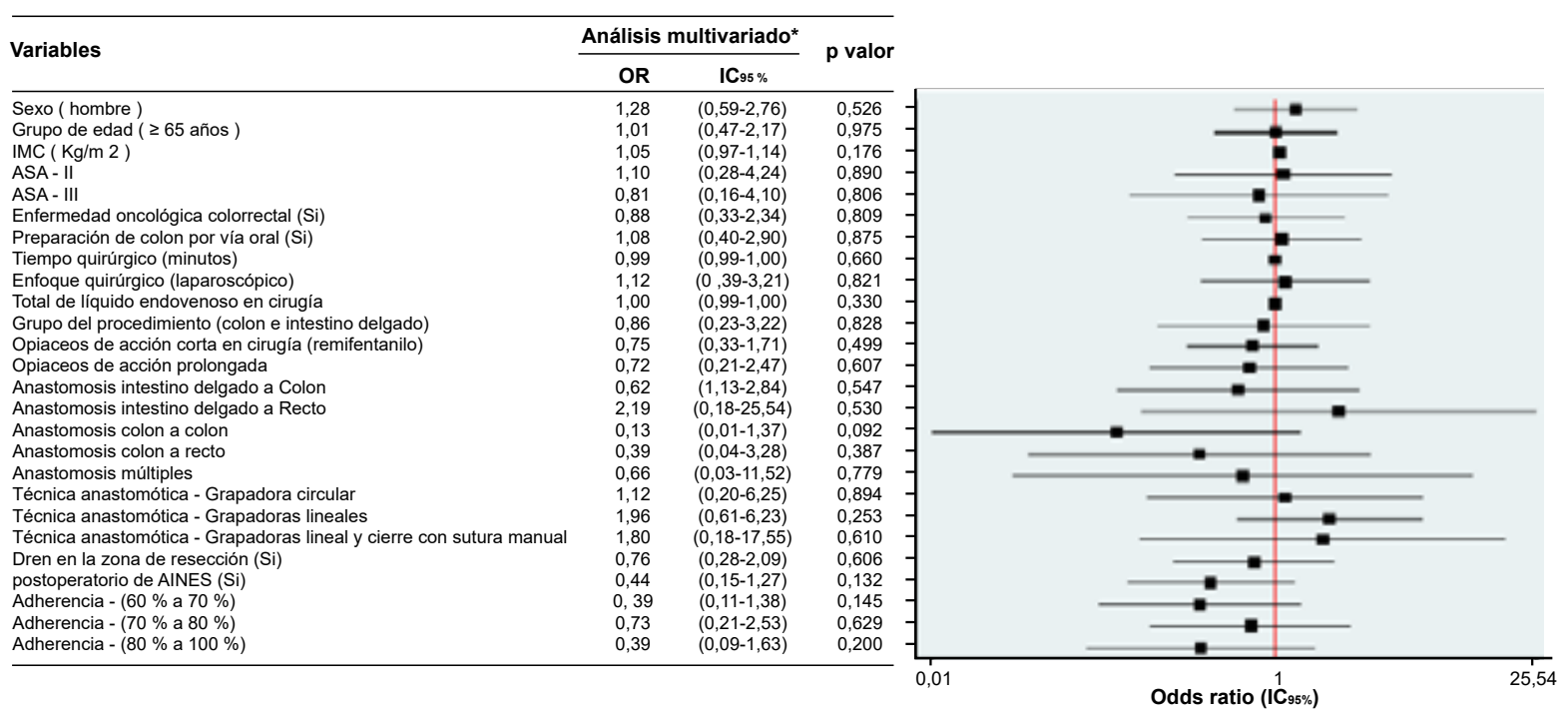

Figura 5. Factores asociados con la aparición de íleo posoperatorio prolongado en cirugía electiva colorrectal con protocolo ERAS. Estudio multicéntrico $(n=648)$ 
equipos ERAS alcancen una curva adecuada de aprendizaje y adherencia al protocolo. Algunos autores mencionan que se requieren mínimo 76 procedimientos de cirugía colorrectal electiva abierta ${ }^{40,41}$. Hasta el momento, la mayoría de los estudios sobre protocolos ERAS y desenlaces en cirugía colorrectal se han centrado en evaluar y comparar el nivel de adherencia al protocolo con resultados como la duración de la estancia hospitalaria posquirúrgica relacionada con el procedimiento principal y frecuencia de complicaciones. Este estudio muestra el análisis de la implementación en instituciones de Latinoamérica y los resultados en cirugía de colon y recto fueron consistentes con los hallazgos positivos reportados en instituciones de países desarrollados 13,31,41-44.

Revisiones sistemáticas recientes han generado la inquietud acerca de no limitar la toma de decisiones al grado de adherencia a las estrategias del programa frente a los desenlaces de interés, sino de conocer el efecto de este conjunto de intervenciones con relación a los desenlaces más críticos ${ }^{19,26}$. Sin embargo, en cirugía de alta complejidad, los resultados demuestran que la parte técnica debe considerarse como el factor determinante para el éxito del procedimiento, estableciéndose que la técnica en la elaboración de las anastomosis, persiste como el mayor factor de riesgo para el fracaso de la misma y debe ser el punto crítico a analizar por los cirujanos, quienes deben buscar estandarizar el procedimiento y definir las herramientas tecnológicas que hacen que el riesgo disminuya para implementar su uso racional. Finalmente, nuestros resultados se pueden considerar como una evaluación del impacto del programa ERAS en hospitales latinoamericanos en los últimos cuatro años.

\section{Conclusiones}

La incidencia global de complicaciones de alto impacto en cirugía electiva de colon y recto (fuga de la anastomosis, complicaciones infecciosas, íleo prolongado y mortalidad) fueron muy bajas tras la implementación del protocolo ERAS en hospitales de Latinoamérica. La adherencia en las estrategias ERAS superior al $80 \%$ (considerada óptima) se identificó como el factor estadísticamente más asociado para disminuir la incidencia de fuga de la anastomosis. Esta adherencia, que impacta positivamente en los desenlaces, ayuda a que disminuyan las fístulas, que representan la complicación más temida por el cirujano, a que pueda ser menor el íleo, que determina la prolongación de la estancia y que sea menor la infección del sitio operatorio (ISO), como indicador de calidad.

Se establece la técnica quirúrgica como el factor determinante del desenlace y que constituye el factor de confusión mayor para el análisis de las estrategias. Las complicaciones durante el postquirúrgico están directamente relacionadas con la capacidad de respuesta funcional del tracto gastrointestinal, sometido a la injuria del procedimiento quirúrgico y a la respuesta sistémica y emocional que genera el procedimiento. Sin embargo, la prehabilitación quirúrgica y rehabilitación durante la fase de recuperación, que se refleja en el grado de adherencia a las estrategias del protocolo ERAS, determinan los resultados de la intervención, lo que indica claramente una relación dosis-respuesta.

\section{Cumplimiento de normas éticas}

Consentimiento informado: En este estudio se hace una revisión retrospectiva de las historias clínicas de los pacientes operados, por lo que no implica riesgo para los pacientes y no requiere la obtención de consentimiento informado. La investigación fue aprobada por el comité de ética institucional (CEIFUS 5I3-I9).

Conflicto de interés: Ninguno por declarar.

Financiación: Trabajo de investigación autofinanciado por los autores.

\section{Referencias}

I. Cone MM, Herzig DO, Diggs BS, Dolan JP, Rea JD, Deveney KE, et al. Dramatic decreases in mortality from laparoscopic colon resections based on data from the Nationwide Inpatient Sample. Arch Surg. 20II;I46:594-9. https://doi.org/IO.IOOI/archsurg.20II.79

2. Callahan MA, Christos PJ, Gold HT, Mushlin AI, Daly JM. Influence of surgical subspecialty training on in-hospital mortality for gastrectomy and colectomy patients. Ann Surg. 2003;238:629-39. https://doi.org/I0.I097/oI.sla.000oo89855.96280.4a 
3. GBD 2017 Causes of Death Collaborators: Roth GA, Abate D, Abate KH, Abay SM, Abbafati C, Abbasi N, et al. Global, regional, and national age-sex-specific mortality for 282 causes of death in 195 countries and territories, 1980-20I7: a systematic analysis for the Global Burden of Disease Study 2017. Lancet. 20I8;392:I736-88. https://doi.org/IO.IOI6/SoI40-6736(I8)32203-7

4. Ljungqvist O, Young-Fadok T, Demartines N. The history of enhanced recovery after surgery and the ERAS society. J Laparoendosc Adv Surg Tech A. 20I7;27:860-2. https://doi.org/IO.IO89/lap.2017.0350

5. Hua CX, Cui YF. [Progress of application for enhanced recovery after surgery in pancreaticoduodenectomy]. Zhonghua Wai Ke Za Zhi. 2016;54:797-8oo.

https://doi.org/I0.3760/cma.j.issn.0529-5815.2016.Io.0I7

6. Straatman J, Cuesta MA, de Lange-de Klerk ESM, van der Peet DL. Hospital cost-analysis of complications after major abdominal surgery. Dig Surg. 2015;32:150-6. https://doi.org/IO.II59/00037186I

7. Lohsiriwat V. Enhanced recovery after surgery vs conventional care in emergency colorectal surgery. World J Gastroenterol. 2014;20:13950-5. https://doi.org/I0.3748/wjg.v20.i38.13950

8. Pedziwiatr M, Wierdak M, Nowakowski M, Pisarska M, Stanek M, Kisielewski M, et al. Cost minimization analysis of laparoscopic surgery for colorectal cancer within the enhanced recovery after surgery (ERAS) protocol: a single-centre, case-matched study. Wideochirurgia Inne Tech Maloinwazyjne. 20I6;II:I4-2I. https://doi.org/I0.5II4/wiitm.2016.586I7

9. Bardram L, Funch-Jensen P, Jensen P, Crawford ME, Kehlet H. Recovery after laparoscopic colonic surgery with epidural analgesia, and early oral nutrition and mobilisation. Lancet. 1995;345:763-4. https://doi.org/IO.IOI6/soI40-6736(95)90643-6

Io. Varadhan KK, Neal KR, Dejong CHC, Fearon KCH, Ljungqvist $\mathrm{O}$, Lobo DN. The enhanced recovery after surgery (ERAS) pathway for patients undergoing major elective open colorectal surgery: A meta-analysis of randomized controlled trials. Clin Nutr. 20I0;29:434-40. https://doi.org/wio.IoI6/j.clnu.20I0.0I.004

II. Zhu D, Wei Y, Ren L, Pan X, Liang L, Zhai S, et al. [Application of enhanced recovery program after surgery (ERAS) in patients undergoing radical resection for colorectal cancer]. Zhonghua Wei Chang Wai Ke Za Zhi. 20I2;15:555-60.

I2. Kehlet H. Accelerated recovery after surgery: A continuous multidisciplinary challenge. Anesthesiology. 2015;123:1219-20. https://doi.org/I0.I097/ALN.0000000000000906

13. Gustafsson UO, Scott MJ, Hubner M, Nygren J, Demartines N, Francis N, et al. Guidelines for perioperative care in elective colorectal surgery: Enhanced recovery after surgery $\left(\mathrm{ERAS}^{\circledR}\right)$ society recommendations: 2018. World J Surg. 2019;43:659-95. https://doi.org/I0.I007/s00268-0I8-4844-y

I4. Kehlet H. Enhanced Recovery After Surgery (ERAS): good for now, but what about the future? Canadian J Anaesth. 2015;62:99-104. https://doi.org/IO.IO07/sI2630-0I4-026I-3

I5. Majumder A, Fayezizadeh M, Neupane R, Elliott HL, Novitsky YW. Benefits of multimodal enhanced recovery pathway in patients undergoing open ventral hernia repair. J Am Coll Surg. 20I6;222:IIO6-I5. https://doi.org/IO.IoI6/j.jamcollsurg.2016.02.015

I6. Oodit RL, Ljungqvist O, Moodley J. Can an Enhanced Recovery After Surgery (ERAS) programme improve colorectal cancer outcomes in South Africa? S Afr J Surg. 20I8;56:8-II.

17. Brescia A, Tomassini F, Berardi G, Sebastiani C, Pezzatini M, Dall'Oglio A, et al. Development of an enhanced recovery after surgery (ERAS) protocol in laparoscopic colorectal surgery: results of the first I2O consecutive cases from a university hospital. Updates Surg. 20I7;69:359-65. https://doi.org/IO.IOO7/sI3304-OI7-0432-I

I8. ERAS-Society. ERAS Guidelines. 2020. Fecha de consulta: 28 de marzo de 2020. Disponible en: http://erassociety.org/

19. Greer NL, Gunnar WP, Dahm P, Lee AE, MacDonald R, Shaukat A, et al. Enhanced recovery protocols for adults undergoing colorectal surgery: A systematic review and meta-analysis. Dis Colon Rectum. 2018;6I:IIO8-I8. https://doi.org/I0.I097/DCR.000000000000II6o

20. Pisarska M, Pędziwiatr M, Małczak P, Major P, Ochenduszko S, Zub-Pokrowiecka A, et al. Do we really need the full compliance with ERAS protocol in laparoscopic colorectal surgery? A prospective cohort study. Int J Surg. 20I6;36:377-82. https://doi.org/Io.IoI6/j.ijsu.20I6.II.o88

2I. Zarate E, Navas A, Barrios A, Sarmiento A, Betancourt $\mathrm{J}$, Mendivelso $\mathrm{F}$, et al. Outcomes in colorectal surgery at reina sofia clinic, Bogota, Colombia, after the implementation of the enhanced recovery after surgery (ERAS) protocol. Clin Nutr ESPEN. 20I8;25:I72. https://doi.org/IO.IoI6/j.clnesp.2018.03.020

22. Mendivelso F, Rodríguez M. Prueba chi-cuadrado de independencia aplicada a tablas $2 x N$. Rev Medica Sanitas. 2018;2I:92-5.

23. Shah PM, Johnston L, Sarosiek B, Harrigan A, Friel CM, Thiele RH, Hedrick TL. Reducing readmissions while shortening length of stay: The positive impact of an enhanced recovery protocol in colorectal surgery. Dis Colon Rectum. 20I7;60:219-27. https://doi.org/IO.I097/DCR.00ooooooooooo748

24. Miller TE, Thacker JK, White WD, Mantyh C, Migaly J, Jin J, et al. Reduced length of hospital stay in colorectal surgery after implementation of an enhanced recovery 
protocol. Anesth Analg. 20I4;II8:I052-6I. https://doi.org/I0.I2I3/ANE.0000000000000206

25. Grieco M, Lorenzon L, Pernazza G, Carlini M, Brescia A, Santoro R, et al. Impact of implementation of the ERAS program in colorectal surgery: a multi-center study based on the "Lazio Network" collective database. Int J Colorectal Dis. 2020;35:445-53. https://doi.org/IO.I007/s00384-019-03496-8

26. Gustafsson UO, Hausel J, Thorell A, Ljungqvist O, Soop M, Nygren J, Enhanced Recovery After Surgery Study Group. Adherence to the enhanced recovery after surgery protocol and outcomes after colorectal cancer surgery. Arch Surg. 20II;I46:57I-7. https://doi.org/IO.IOoI/archsurg.20IO.309

27. Arrick L, Mayson K, Hong T, Warnock G. Enhanced recovery after surgery in colorectal surgery: Impact of protocol adherence on patient outcomes. J Clin Anesth. 2019;55:7-I2. https://doi.org/IO.IoI6/j.jclinane.20I8.I2.034

28. Giaccaglia V, Salvi PF, Antonelli MS, Nigri G, Pirozzi F, Casagranda B, et al. Procalcitonin reveals early dehiscence in colorectal surgery: The PREDICS study. Ann Surg. 20I6;263:967-72.

https://doi.org/ I0.I097/SLA.0000000000001365

29. Nikolian VC, Kamdar NS, Regenbogen SE, Morris AM, Byrn JC, Suwanabol PA, et al. Anastomotic leak after colorectal resection: A population-based study of risk factors and hospital variation. Surgery. 2017;16I:I6I9-27. https://doi.org/IO.IOI6/j.surg.2016.12.033

30. ERAS Compliance Group. The impact of enhanced recovery protocol compliance on elective colorectal cancer resection: Results from an international registry. Ann Surg. 2015;26I:II53-9. https://doi.org/I0.I097/SLA.000000000000I029

3I. Pedziwiatr M, Pisarska M, Kisielewski M, Matlok M, Major P, Wierdak M, et al. Is ERAS in laparoscopic surgery for colorectal cancer changing risk factors for delayed recovery? Med Oncol. 20I6;33:25. https://doi.org/IO.IOO7/sI2032-0I6-0738-8

32. Ripolles-Melchor J, Ramirez-Rodriguez JM, Casans-Francés R, Aldecoa C, Abad-Motos A, Logroño-Egea $\mathrm{M}$, et al. Association between use of enhanced recovery after surgery protocol and postoperative complications in colorectal surgery: The postoperative outcomes within enhanced recovery after surgery protocol (POWER) study. JAMA Surg. 2019;154:725-36. https://doi.org/IO.IOOI/jamasurg.20I9.0995

33. Mata J, Fiore JF, Pecorelli N, Stein BL, Liberman S, Charlebois P, Feldman LS. Predictors of adherence to enhanced recovery pathway elements after laparoscopic colorectal surgery. Surg Endosc. 2018;32:1812-9. https://doi.org/IO.IOO7/s00464-0I7-5865-6

34. Grant MC, Pio Roda CM, Canner JK, Sommer P, Galante D, Hobson D, et al. The impact of anesthesia-influenced process measure compliance on length of stay:
Results from an enhanced recovery after surgery for colorectal surgery cohort. Anesth Analg. 2019;128:68-74. https://doi.org/IO.I2I3/ANE.00o0ooooooo03458

35. Pedziwiatr M, Pisarska M, Kisielewski M, Major P, Mydlowska A, Rubinkiewicz M, et al. ERAS protocol in laparoscopic surgery for colonic versus rectal carcinoma: are there differences in short-term outcomes? Med Oncol. 20I6;33:56. https://doi.org/IO.IOO7/sI2032-0I6-0772-6

36. Ahmed J, Khan S, Lim M, Chandrasekaran TV, MacFie J. Enhanced recovery after surgery protocols - compliance and variations in practice during routine colorectal surgery. Colorectal Dis. 20I2;I4:I045-5I. https://doi.org/IO.IIII/j.I463-I3I8.20II.02856.x

37. Pedrazzani C, Conti C, Turri G, Lazzarini E, Tripepi M, Scotton G, et al. Impact of age on feasibility and shortterm outcomes of ERAS after laparoscopic colorectal resection. World J Gastrointest Surg. 20I9;II:395-406. https://doi.org/IO.4240/wjgs.viI.iIo.395

38. Ban KA, Berian JR, Ko CY. Does implementation of enhanced recovery after surgery (ERAS) protocols in colorectal surgery improve patient outcomes? Clin Colon Rectal Surg. 2019;32:I09-II3. https://doi.org/IO.IO55/s-0038-I676475

39. Barbieux J, Hamy A, Talbot MF, Casa C, Mucci S, Lermite E, Venara A. Does enhanced recovery reduce postoperative ileus after colorectal surgery? J Visc Surg. 2017;154:79-85. https://doi/I0.Iol6/j.jviscsurg.2016.08.003.

40. Lohsiriwat V. Learning curve of enhanced recovery after surgery program in open colorectal surgery. World J Gastrointest Surg. 20I9;II:I69-78. https://doi.org/IO.4240/wjgs.vir.i3.I69

4I. van Zelm R, Coeckelberghs E, Sermeus W, De Buck van Overstraeten A, Weimann A, Seys D, et al. Variation in care for surgical patients with colorectal cancer: protocol adherence in I2 European hospitals. Int J Colorectal Dis. 20I7;32:147I-8. https://doi.org/IO.IO07/s00384-0I7-2863-Z

42. Gustafsson UO, Oppelstrup H, Thorell A, Nygren J, Ljungqvist $\mathrm{O}$. Adherence to the ERAS protocol is associated with 5-year survival after colorectal cancer surgery: A retrospective cohort study. World J Surg. 20I6;40:I74I-7. https://doi.org/I0.I007/soo268-0I6-3460-y

43. Grant MC, Hanna A, Benson A, Hobson D, Wu CL, Yuan CT, et al. Dedicated operating room teams and clinical outcomes in an enhanced recovery after surgery pathway for colorectal surgery. J Am Coll Surg. 2018;226:267-76. https://doi.org/IO.IoI6/j.jamcollsurg.20I7.I2.oIO

44. Pędziwiatr M, Pisarska M, Major P, Grochowska A, Matłok M, Przęczek K, et al. Enhanced Recovery after Surgery protocol (ERAS) combined with laparoscopic colorectal surgery diminishes the negative impact of sarcopenia on short-term outcomes. Clin Nutr ESPEN. 20I6;I2:e49. https://doi.org/IO.IoI6/j.clnesp.20I6.02.06I 